\title{
Influenza A virus antibodies show no association with pancreatic islet autoantibodies in children genetically predisposed to type 1 diabetes
}

\author{
Anita Kondrashova ${ }^{1}$ - Noora Nurminen ${ }^{1}$ Maarit Patrikainen ${ }^{1} \cdot$ Heini Huhtala ${ }^{2}$. \\ Jussi Lehtonen $^{1}$ - Jorma Toppari ${ }^{3,4}$. Jorma Ilonen ${ }^{5,6}$ - Olli G. Simell ${ }^{4}$ - Riitta Veijola ${ }^{7}$. \\ Mikael Knip ${ }^{8,9,10,11} \cdot$ Heikki Hyöty ${ }^{1,12}$
}

Received: 8 May 2015 / Accepted: 21 July 2015 / Published online: 8 August 2015

(C) Springer-Verlag Berlin Heidelberg 2015

\begin{abstract}
Aims/hypothesis Viral infections have long been considered potential triggers of beta cell autoimmunity and type 1 diabetes. Recent studies have suggested that influenza A virus might increase the risk of type 1 diabetes. The present study evaluates this risk association in prospectively observed children at the time when islet autoimmunity starts and autoantibodies are first detected.

Methods IgG class antibodies to influenza A virus were analysed in 95 case children whose antibody screening test turned permanently positive for two or more islet
\end{abstract}

Heikki Hyöty

Heikki.Hyoty@uta.fi

1 School of Medicine, University of Tampere, Biokatu 10, FIN-33520 Tampere, Finland

2 School of Health Sciences, University of Tampere, Tampere, Finland

3 Department of Physiology, University of Turku, Turku, Finland

4 Department of Pediatrics, Turku University Hospital, Turku, Finland

5 Department of Clinical Microbiology, University of Eastern Finland, Kuopio, Finland

6 Immunogenetics Laboratory, University of Turku, Turku, Finland

7 Department of Pediatrics, University of Oulu, Oulu, Finland

8 Children's Hospital, University of Helsinki and Helsinki University Hospital, Helsinki, Finland

9 Research Programs Unit, Diabetes and Obesity, University of Helsinki, Helsinki, Finland

10 Folkhälsan Research Center, Helsinki, Finland

11 Tampere Center for Child Health Research, Tampere University Hospital, Tampere, Finland

12 Fimlab Laboratories, Pirkanmaa Hospital District, Tampere, Finland autoantibodies and from 186 autoantibody-negative and non-diabetic control children who were matched for time of birth, sex, date of sampling and HLA-conferred risk of diabetes in the Finnish Type 1 Diabetes Prediction and Prevention (DIPP) study. Virus antibodies were measured from the first autoantibody-positive sample using an enzyme immunoassay. None of the children had been vaccinated against influenza A. Results The prevalence of influenza A virus antibodies did not differ between the case and control children ( $42 \%$ vs $38 \%$; $p=0.392$ ) and the median antibody levels were also comparable in the two groups (3.0 vs 3.8 enzyme immunoassay units). A similar result was obtained when case and control children were compared separately in subgroups according to different sex, age and HLA-DQ genotype. However, girls had higher antibody levels than boys among both case and control children (median antibody levels 9.0 vs 2.3 enzyme immunoassay units; $p=0.01$ ). Conclusions/interpretation Our results suggest that influenza A infections are not associated with the development of islet autoimmunity in young children with increased genetic susceptibility to type 1 diabetes.

Keywords Influenza A virus · Type 1 diabetes · Virus antibodies

\begin{abstract}
Abbreviations
DIPP Diabetes Prediction and Prevention

EIU Enzyme immunoassay unit
\end{abstract}

\section{Introduction}

Both genetic and environmental factors contribute to the pathogenesis of type 1 diabetes. Immune-mediated mechanisms 
are considered to be important and autoantibodies against beta cell proteins predict clinical disease quite reliably [1]. Virus infections have long been cited as potential triggers of the process. Currently, enterovirus infections are considered as one of the main viral candidates but other viruses may also play a role [2].

Recent studies have suggested that influenza A virus infections might increase the risk of type 1 diabetes. A higher number of new cases with type 1 diabetes occurred soon after the swine flu H1N1 pandemic [3, 4]. Human and avian influenza A viruses can also infect human pancreatic cell lines and human pancreatic islets, and cause pancreatitis and diabetes in animals [5, 6]. Moreover, the incidence of narcolepsy, an autoimmune disease caused by selective destruction of orexinproducing neurons in the central nervous system, increased a few months after the pandemic AS03-adjuvanted H1N1 vaccination in 2010 in Finland, Sweden and Ireland [7]. The mechanisms of this risk association are not completely understood but both adjuvant- and virus-induced immune responses could be involved [7]. At the same time, narcolepsy also increased in northern China where the vaccine was not used, suggesting that $\mathrm{H} 1 \mathrm{~N} 1$ infection itself could trigger narcolepsy [8]. These findings support the hypothesis that influenza A virus has the potential to induce autoimmune reactions.

The current study was designed to evaluate the association between influenza A virus infections and type 1 diabetes in a case-control study nested in a prospective cohort of young children who have been followed from birth. Study of this cohort made it possible to test whether these viruses play a role in the initiation of processes that damage beta cells.

\section{Methods}

Study participants The study children participated in the Diabetes Prediction and Prevention (DIPP) birth cohort study in Finland. In this study, all newborn infants born at three University hospitals in Finland are first screened for $H L A-D Q B 1$ alleles that affect the risk of developing type 1 diabetes, and the children with increased HLA-defined risk are enrolled in the follow-up as described previously [9]. The follow-up includes visits to the study clinic every 3-12 months when serum samples are taken for the screening of disease-associated islet autoantibodies (islet cell antibodies, antibodies to insulin, glutamic acid decarboxylase 65 and the protein tyrosine phosphatase-related IA-2 antigen). Serum samples have been stored frozen at $-70^{\circ} \mathrm{C}$.

The case children included all 95 children who had turned positive for two or more of the autoantibodies associated with type 1 diabetes (54 had also developed clinical type 1 diabetes) in the Tampere DIPP centre and regularly followed from birth. They were born between 1997 and 2007 (67\% were boys); the median age at the first detection of autoantibodies was 3.0 years (range 1.4-8.1 years) and at the diagnosis of type 1 diabetes was 6.3 years (range 1.4-13.2 years). The first autoantibodypositive samples were selected for the analysis of influenza $\mathrm{A}$ virus antibodies. One to two autoantibody-negative nondiabetic control participants $(n=186)$ were selected pairwise for each case child and matched for calendar time of birth ( \pm 1 month), sex, date of sampling ( \pm 1 month) and HLA-defined risk of type 1 diabetes. No case or control participants were from birth cohorts that received influenza A vaccination in the national programme and no influenza vaccinations were reported in questionnaires during the follow-up.

All children had written parental consent and the Ethics Committee of Tampere University Hospital had granted approval for the DIPP study. The study was carried out in accordance with the principles of the Declaration of Helsinki.

Antibody assays Serum $\mathrm{IgG}$ class antibodies to influenza A virus were analysed using an enzyme immunoassay. The wells of a microtitre plate (Immunoplate II; Nunc, Roskilde, Denmark) were coated overnight at room temperature with $50 \mu \mathrm{l}$ of influenza A antigen $(3 \mu \mathrm{g} / \mathrm{ml}$ per well of concentrated viral matrix and nucleoproteins of Beijing strain; Virion Serion, Wurzburg, Germany; product code BA1231VS), then washed four times with washing buffer (PBS $+0.5 \%$ Tween) and blocked by $0.1 \%$ BSA in PBS for $30 \mathrm{~min}$ at room temperature. Serum was incubated for $1 \mathrm{~h}$ at $37^{\circ} \mathrm{C}$ in $1 / 200$ dilution in $\mathrm{PBS}+1 \% \mathrm{BSA}+0.5 \mathrm{~mol} / \mathrm{l} \mathrm{NaCl}+0.05 \%$ Tween 20 , followed by washings and incubation of polyclonal rabbit anti-human IgG conjugate (P0214, Dako, Glostrup, Denmark) in $1 / 3,000$ dilution for $30 \mathrm{~min}$ at $37^{\circ} \mathrm{C}$. Finally, tetramethylbenzidine substrate (OPD-tablet, citrate buffer, $\mathrm{H}_{2} \mathrm{O}_{2}$ ) was added and allowed to react for $15 \mathrm{~min}$ in room temperature. The reaction was stopped by adding $100 \mu \mathrm{l} /$ well of $1 \mathrm{~N} \mathrm{H}_{2} \mathrm{SO}_{4}$, and the results were read at $490 \mathrm{~nm}$ in a spectrophotometer (Victor ${ }^{2}$ Perkin Elmer Wallac 1420 Multilabel Counter, Waltham, Massachusetts, USA). Samples from case and corresponding control children were tested blindly in the same test run. The antibody results are given as EIU (enzyme immunoassay units), which are calculated using the following formula: (absorbance of the tested sample - absorbance of negative control sample)/(absorbance of positive control sample - absorbance of negative control sample) $\times 100$. An EIU value of 10 was used as a cut-off for seropositivity. The assay conditions were optimised using paired sera from ten patients with confirmed acute influenza A virus infection (collaboration with T. Ziegler, National Institute for Health and Welfare, Helsinki, Finland). These sera showed a clear increase in median IgG EIU levels between acute and convalescent sera (from 52 to $170 \mathrm{EIU}$; $p<0.001)$.

Statistical analysis IgG prevalence in cases and controls was analysed using conditional logistic regression (Stata 12.1. 
a

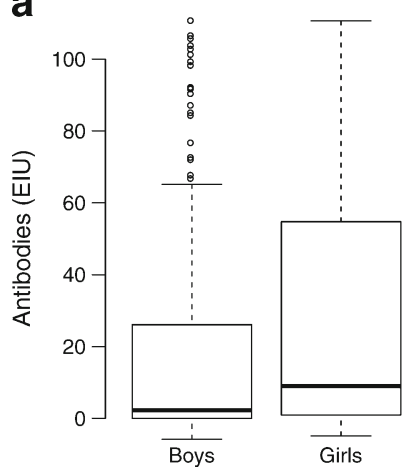

b

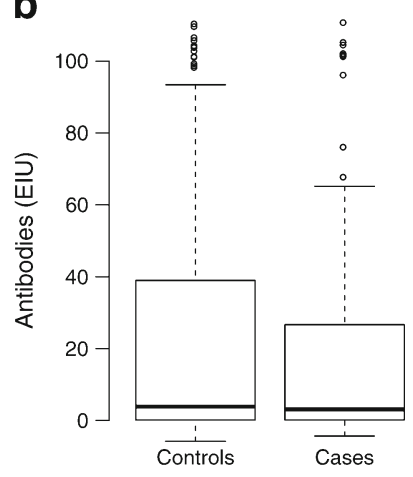

Fig. 1 (a) Levels of IgG class antibodies to influenza A virus in boys and girls $(p=0.01)$, and (b) in children who turned positive for multiple autoantibodies associated with type 1 diabetes, as a marker of the subclinical autoimmune process leading to type 1 diabetes (cases), and in their matched controls $(p=0.356)$. Each box plot represents the median (horizontal line), interquartile range (box) and the lowest and highest values (error bars). The outliers are presented as circles

statistical program, StataCorp, College Station, Texas, USA, and $\mathrm{R}$ version 3.1.2., https://www.r-project.org/). A $p$ value of less than 0.05 was set as an indicator of statistical significance. Sample size was estimated by a priori power calculations based on results from previous publications.

\section{Results}

The prevalence of influenza A virus IgG increased by age from $24 \%$ among children aged 3 years or less to $53 \%$ among older children $(p<0.0001)$. Girls had a higher antibody prevalence than boys $(48 \%$ vs $35 \%, p=0.04)$ and their median antibody levels were higher (9.0 EIU vs $2.3 \mathrm{EIU}, p=0.01$; Fig. 1a). The antibody prevalence did not differ between children carrying moderate/low-risk HLA-DQ genotypes and those with the high-risk genotypes ( $46 \%$ vs $38 \% ; p=0.30$ ). Antibody levels showed a seasonal pattern being highest in

samples collected during the second and third quarters of the year (median EIU 14 in April to June and 9 in July to September) and lowest in the last and first quarters of the year (median EIU 2 and 3, respectively; $p=0.06$ ).

The antibody prevalence did not differ between case and control children ( $42 \%$ vs $38 \%$; Table 1$)$ and the antibody levels were comparable between the two groups (median 3.0 vs 3.8 EIU; Fig. 1b). The same was true in subgroups separated according to different age, sex, HLA-DQ genotype or progression to clinical type 1 diabetes (Table 1 ). When only seropositive children were included in this comparison, the average antibody levels were lower in cases than in controls (median 36.1 vs $61.5 \mathrm{EIU} ; p=0.015$ ).

\section{Discussion}

The present study is the first carried out among prospectively observed children to evaluate the association between influenza A virus infections and the initiation of islet autoimmunity. Influenza A virus antibodies were frequent, suggesting that these infections are common in young children, which is in line with the findings of previous studies $[10,11]$. The fact that antibodies did not differ between case and control children suggests that influenza A virus infections are not associated with the initiation of islet autoimmunity. All case children had developed multiple islet autoantibodies and many had also progressed to clinical diabetes, indicating that they were truly affected by the beta cell damaging process [1].

Girls were more frequently positive for influenza A antibodies than boys, and their average antibody levels were also higher. Previous studies have shown that girls produce stronger immune responses to several viruses $[12,13]$. Thus, our observation may reflect a difference in immune responsiveness to influenza A virus rather than in the number of infections per se. Cases and controls were matched for sex,

Table 1 Prevalence of influenza A virus antibodies in children who developed islet autoimmunity and type 1 diabetes compared with nondiabetic autoantibody-negative control children

\begin{tabular}{|c|c|c|c|c|c|}
\hline \multirow[t]{2}{*}{ Group } & \multicolumn{2}{|c|}{ Prevalence of influenza A virus antibodies } & \multirow[t]{2}{*}{ OR } & \multirow[t]{2}{*}{$95 \% \mathrm{CI}$} & \multirow[t]{2}{*}{$p$ value } \\
\hline & Cases $n+/ n(\%)$ & Controls $n+/ n(\%)$ & & & \\
\hline All & $40 / 95(42)$ & $70 / 186(38)$ & 1.3 & $0.7,2.2$ & 0.392 \\
\hline Cases who progressed to type 1 diabetes & $19 / 54(35)$ & $34 / 106(32)$ & 1.2 & $0.6,2.5$ & 0.640 \\
\hline Boys & $23 / 62(37)$ & $41 / 123(33)$ & 1.2 & $0.6,2.4$ & 0.570 \\
\hline Girls & $17 / 33(52)$ & $29 / 63(46)$ & 1.3 & $0.5,3.4$ & 0.605 \\
\hline Age $\leq 3$ years & $8 / 34(24)$ & $12 / 65(19)$ & 1.1 & $0.45,3.2$ & 0.925 \\
\hline Age $>3$ years & $32 / 61(53)$ & $58 / 121(48)$ & 1.3 & $0.7,2.5$ & 0.498 \\
\hline High-risk HLA-DQ & $30 / 75(40)$ & $54 / 149(36)$ & 1.3 & $0.7,2.4$ & 0.479 \\
\hline Moderate-risk/low-risk HLA-DQ & $10 / 20(50)$ & $16 / 37(43)$ & 1.3 & $0.4,4.2$ & 0.626 \\
\hline
\end{tabular}

Antibodies were analysed from the first autoantibody-positive sample 
eliminating the possibility that this phenomenon could have biased the comparison between cases and controls.

One of the virtues of the present study is that none of the children had received influenza A vaccinations. This allowed us to exclude the potential biasing effect of vaccine-related immune responses. All children were also older than 1 year at sampling, which excluded any interference with maternal virus antibodies in the antibody assays. In addition, virus antibodies were analysed when children had normal glucose tolerance, and therefore metabolic disturbances could not have affected antibody responses. Importantly, the cases and controls were also matched for HLA genotype, which may modulate antibody responses.

The present study also has some limitations. Since virus antibodies were measured from the first autoantibodypositive sample we were not able to address the possible effect of influenza virus infections on the progression from beta cell autoimmunity to clinical disease. Furthermore, the study covered only one geographical area and population, and it is possible that viruses that circulate at certain other times and places may have a diabetogenic effect. For example, none of the children had experienced the H1N1 pandemic in 2009-2010.

In conclusion, our results suggest that seasonal influenza A infections are not associated with the development of islet autoimmunity in children genetically susceptible to type 1 diabetes. However, we cannot exclude the possibility that certain influenza virus strains, such as the H1N1 swine flu epidemic strain, could play a role in the diabetic disease process.

Acknowledgements We thank E. Jalonen (Department of Virology, School of Medicine, University of Tampere, Tampere, Finland) and E. Korhonen (Tampere Centre for Child Health Research, Tampere University Hospital, Tampere, Finland) for their skilful assistance in laboratory work and data management. We also thank all children and their parents participating in the DIPP study.

Funding This study was supported by the Diabetes Research Foundation in Finland, Sigrid Juselius Foundation, Competitive Research Funding of the Tampere University Hospital, the Juvenile Diabetes Research Foundation (JDRF), the Academy of Finland and the Päivikki and Sakari Sohlberg's Foundation.

Duality of interest The authors declare that there is no duality of interest associated with this manuscript.

Contribution statement AK contributed to the design, analysis, interpretation of data and manuscript drafting. NN, HHu, MP and JL contributed to acquisition and analysis of data and revising the article. $\mathrm{HHy}$ contributed to conception and design, analysis and interpretation of data and revising the manuscript. JI, MK, RV, OGS, JT and HHy are DIPP Principal Investigators and MK is additionally in charge of the Tampere DIPP centre, all contributed to the conception and design as well as revising the article. All authors read and approved the final version of the paper. HHy is the guarantor of this work.

\section{References}

1. Ziegler AG, Rewers M, Simell O et al (2013) Seroconversion to multiple islet autoantibodies and risk of progression to diabetes in children. JAMA 309:2473-2479

2. Nurminen N, Oikarinen S, Hyöty H (2012) Virus infections as potential targets of preventive treatments for type 1 diabetes. Rev Diabet Stud 9:260-271

3. Nenna R, Papoff P, Moretti C et al (2011) Detection of respiratory viruses in the 2009 winter season in Rome: 2009 influenza A (H1N1) complications in children and concomitant type 1 diabetes onset. Int J Immunopathol Pharmacol 24:651-659

4. Valdes C, Unanue N, Hernandez M et al (2013) Is there a link between influenza and type I diabetes? Increased incidence of TID during the pandemic H1N1 influenza of 2009 in Chile. Pediatr Endocrinol Rev 11:161-166

5. Capua I, Mercalli A, Pizzuto MS et al (2013) Influenza A viruses grow in human pancreatic cells and cause pancreatitis and diabetes in an animal model. J Virol 87:597-610

6. Lipatov AS, Kwon YK, Pantin-Jackwood MJ, Swayne DE (2009) Pathogenesis of H5N1 influenza virus infections in mice and ferret models differs according to respiratory tract or digestive system exposure. J Infect Dis 199:717-725

7. Partinen M, Kornum BR, Plazzi G, Jennum P, Julkunen I, Vaarala O (2014) Narcolepsy as an autoimmune disease: the role of H1N1 infection and vaccination. Lancet Neurol 13:600-613

8. Han F, Lin L, Warby SC et al (2011) Narcolepsy onset is seasonal and increased following the 2009 H1N1 pandemic in China. Ann Neurol 70:410-417

9. Näntö-Salonen K, Kupila A, Simell S et al (2008) Nasal insulin in prevention of type 1 diabetes in children with genetic risk and autoantibodies. Lancet 372:1746-1755

10. Bodewes R, de Mutsert G, van der Klis FR et al (2011) Prevalence of antibodies against seasonal influenza $\mathrm{A}$ and $\mathrm{B}$ viruses in children in Netherlands. Clin Vaccine Immunol 18:469-476

11. Sauerbrei A, Langenhan T, Brandstadt A et al (2014) Prevalence of antibodies against influenza $\mathrm{A}$ and $\mathrm{B}$ viruses in children in Germany, 2008 to 2010. Euro Surveill 19:20687

12. Muenchoff M, Goulder PJ (2014) Sex differences in pediatric infectious diseases. J Infect Dis 209(Suppl 3):S120-126

13. Fischer J, Jung N, Robinson N, Lehmann C (2015) Sex differences in immune responses to infectious diseases. Infection. doi:10.1007/ s15010-015-0791-9 